\title{
Magnetic resonance spectroscopy and gadolinium enhancement assist in the diagnosis of nonalcoholic Marchiafava-Bignami disease with necrosis lesions: a case description
}

\author{
Zhiwei Zhou ${ }^{1,2 \#} \wedge$, Qinghui $\mathrm{Li}^{3 \#}$, Chengyu Pan ${ }^{2}$, Tao Liang ${ }^{1,2}$, Tijiang Zhang ${ }^{3}$, Ping Xu ${ }^{1,2} \wedge$ \\ ${ }^{1}$ Soochow University Medical College, Suzhou, China; ${ }^{2}$ Department of Neurology, Affiliated Hospital of Zunyi Medical University, Zunyi, China; \\ ${ }^{3}$ Department of Radiology, Affiliated Hospital of Zunyi Medical University, Medical Imaging Center of Guizhou Province, Zunyi, China
}

\#These authors contributed equally to this work.

Correspondence to: Ping Xu. Soochow University Medical College, Suzhou, China; Department of Neurology, Affiliated Hospital of Zunyi Medical University, Zunyi, China. Email: xuping527@vip.sina.com; Tijiang Zhang, Department of Radiology, Affiliated Hospital of Zunyi Medical University, Medical Imaging Center of Guizhou Province, Zunyi, China. Email: tijzhang@163.com.

Submitted Jun 15, 2021. Accepted for publication Jul 26, 2021.

doi: 10.21037/qims-21-632

View this article at: https://dx.doi.org/10.21037/qims-21-632

\section{Introduction}

Marchiafava-Bignami disease (MBD) is a severe neuropsychiatric disorder characterized by demyelination, necrosis, and cystic changes of the corpus callosum (CC) $(1,2)$. Common symptoms include an altered mental state, impaired memory, disorientation, and seizures (1). MBD has been frequently associated with chronic alcoholism in the literature, and is easily overlooked and misdiagnosed in an environment of other malnutrition predisposing conditions, such as gastritis and anemia (3). Noncontrast head computerized tomography has revealed symmetrical hypodensity lesions of the CC, and cerebral magnetic resonance imaging (MRI) has shown symmetrical hyperintensity of the CC on T2-weighted images (T2WI) and fluid-attenuated inversion recovery (FLAIR) images, and hypointensity on T1-weighted images (T1WI), with or without extra-callosal lesions (1). CC lesions of MBD do not have a mass effect, and there is a classic sandwich sign due to central demyelination or necrosis of the CC and sparing of the dorsal and ventral layers (4). The CC lesions eventually improve completely or become wellcircumscribed cavitations (4).

The diagnosis of MBD requires a combination of medical history, clinical manifestations, and neuroimaging, especially cerebral MRI findings (5). A differentiated diagnosis from glioblastoma $(\mathrm{GBM})$ is required in cases of MBD with necrosis and cystic degeneration in the CC. Herein, we present the first case of magnetic resonance spectroscopy (MRS) and gadolinium enhancement being applied, which provided additional information to assist in the diagnosis of nonalcoholic MBD with necrosis lesions in the CC, thus making it possible to differentiate from GBM.

\section{Case presentation}

A 64-year-old male was admitted with memory deficit for 1 year, and without headache, dizziness, unconsciousness, seizures, or paresthesia. The patient developed hypodynamia for 6 months (which was obvious in the right arm); he could walk independently and had not suffered weight loss. The patient had been a smoker for more than 40 years but had given up smoking for half a year, and had drunk occasionally but had been abstinent for 30 years. He had a history of intermittent stomachache for 4 years, and gastroscopy showed chronic gastritis. He received blood transfusion due to anemia 1 month prior to admission.

^ ORCID: Zhiwei Zhou, 0000-0001-5499-8740; Ping Xu, 0000-0002-4118-2598. 
On admission, he was conscious and had a slow response, dyscalculia, memory impairment, and disorientation. His muscle strength was $4 / 5$ in both upper extremities and $5 / 5$ in both lower extremities [according to the UK Medical Research Council (MRC) grading] with normal muscle tone. The right biceps and triceps were hyperreflexia, with a positive bilateral Babinski sign with dorsiflexion.

Laboratory abnormalities included the following: red blood cell (RBC) count, $2.77 \times 10^{12} / \mathrm{L}$ (normal range, 4.3-5.8); hemoglobin, $66.0 \mathrm{~g} / \mathrm{L}(130-175 \mathrm{~g} / \mathrm{L})$; hematocrit, $0.22 \mathrm{~L} / \mathrm{L}$ (0.4-0.5 L/L); mean corpuscular volume, $78.0 \mathrm{fL}(80$ $100 \mathrm{fL})$; mean corpuscular hemoglobin, $23.8 \mathrm{pg}(27-34 \mathrm{pg})$; mean corpuscular hemoglobin concentration, $306 \mathrm{~g} / \mathrm{L}$ (316-354 g/L); RBC volume distribution width, $21.6 \%$ (11.5-14.5\%); ferritin, $8.3 \mu \mathrm{g} / \mathrm{L}(23.9-336.2 \mu \mathrm{g} / \mathrm{L})$; albumin, $32.0 \mathrm{~g} / \mathrm{L}(40-55 \mathrm{~g} / \mathrm{L})$; and proalbumin, $125 \mathrm{mg} / \mathrm{L}(200-$ $400 \mathrm{mg} / \mathrm{L})$. Digestive tract tumor markers, coagulation tests, renal function, myocardial enzyme, electrolyte, blood glucose, and cholesterol were normal, and HIV, and syphilis antibodies were negative. Cerebrospinal fluid (CSF) studies were unremarkable, and electrocardiogram was normal.

Cranial MRI (Figure 1) showed symmetrically distributed lesions in the white matter and CC, with hypointensity on T1WI and hyperintensity on T2WI, as well as necrosis and cystic degeneration with well-defined margins in the center of the lesions in the genu, body, and splenium of the CC (Figure 1A-1G). The central part of the lesions in the genu, body, and splenium of the CC showed hypointensity on diffusion-weighted imaging (DWI), while the peripheral part of the lesions showed hyperintensity on DWI (Figure $1 H, I)$. Magnetic resonance angiography showed no abnormalities in the intracranial arteries. The diagnosis of MBD was made based on the following clinical, laboratory, and radiological findings: (I) a history of malnutrition; (II) with neuropsychiatric symptoms (memory deficit); (III) symmetrical lesions of the CC and white matter with hypointensity on T1WI and hyperintensity on T2WI (thereby excluding other diseases including Wernicke encephalopathy and reversible splenial lesion syndrome) and magnetic resonance angiography showing no abnormalities in the intracranial arteries (thereby excluding cerebrovascular diseases); and (IV) unremarkable CSF studies (thereby excluding central nervous system infections).

However, a differentiated diagnosis from GBM was required due to necrosis and cystic degeneration in the CC. Additionally, gadolinium enhancement and MRS were performed. No abnormal contrast enhancement was observed (Figure 1L-1Q). The choline (Cho)/ creatine $(\mathrm{Cr})$ and $\mathrm{N}$-acetylaspartate $(\mathrm{NAA}) / \mathrm{Cr}$ ratios on the MRS of the central and peripheral parts of the lesion in the splenium were 1.03 and 0.469 , and 1.27 and 1.46, respectively (Figure $1 R-1 U$ ). These MRS findings suggested nonneoplastic lesions, which supported the final diagnosis of MBD. The patient was treated with intramuscular thiamine $(100 \mathrm{mg} /$ day $)$ and mecobalamin $(0.5 \mathrm{mg} / \mathrm{day})$, and pantoprazole sodium was used for gastritis. For anemia, hypoferric anemia was considered, and iron sucrose $(100 \mathrm{mg})$ was administered intravenously twice a week. Repeated blood routine and biochemical tests were performed 7 days after admission and revealed the following: RBC count, $3.19 \times 10^{12} / \mathrm{L}$; hemoglobin, $75.0 \mathrm{~g} / \mathrm{L}$; hematocrit, $0.26 \mathrm{~L} / \mathrm{L}$; mean corpuscular hemoglobin, $23.5 \mathrm{pg}$; mean corpuscular hemoglobin concentration, $291 \mathrm{~g} / \mathrm{L}$; RBC volume distribution width, $20.7 \%$; albumin, $35.1 \mathrm{~g} / \mathrm{L}$; and proalbumin, $108 \mathrm{mg} / \mathrm{L}$. No obvious abnormality was observed in the other items. After 10 days of treatment, his memory deficit was hardly improved. Treatment with daily oral thiamine $(30 \mathrm{mg})$ and vitamin B12 $(50 \mu \mathrm{g})$ continued after discharge.

This study conformed to the provisions of the Declaration of Helsinki (as revised in 2013), and the clinical data collection of this patient was approved by the institutional research committee. Written informed consent was obtained from the patient.

\section{Discussion}

Hundreds of MBD cases have been reported worldwide, and the occurrence of MBD has exhibited an increasing trend (6). Although most previous studies have reported MBD in chronic alcoholics, several studies have reported MBD in patients with malnutrition, such as those with anorexia nervosa and vegetarians $(7,8)$. Given our patient's brief exposure to alcohol and subsequent abstinence, we believed that his MBD was triggered by poor nutrition (gastritis and hypoferric anemia). Unfortunately, at present, there are no management guidelines for MBD.

According to the time interval between the onset of symptoms and hospital admission, MBD is divided into acute ( $\leq 2$ weeks), subacute ( $>2$ weeks), and chronic ( $>3$ months) phases (1). The delay from the onset of symptoms to hospital admission in our patient was $>1$ year, which belongs to the chronic phase. Nevertheless, the MRI findings of our patient revealed two stages: chronic lesions in the center of the CC and subacute lesions in the peripheral part. Similarly, Quintas-Neves et al. also reported an MBD 

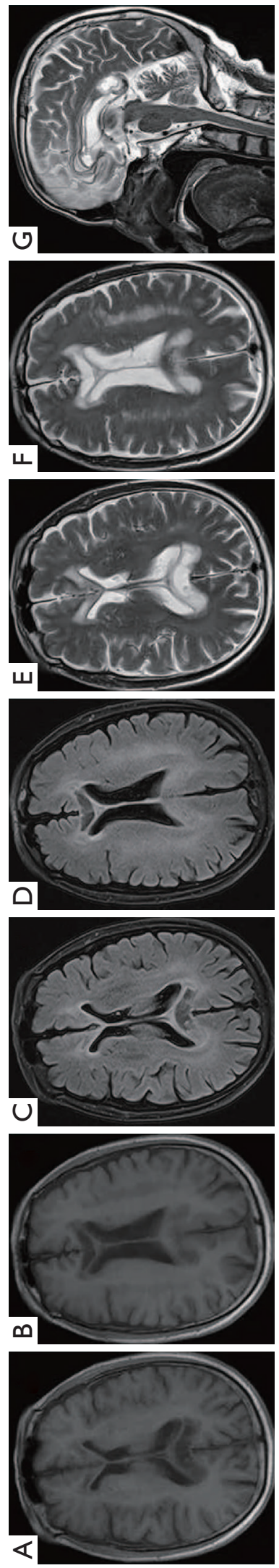
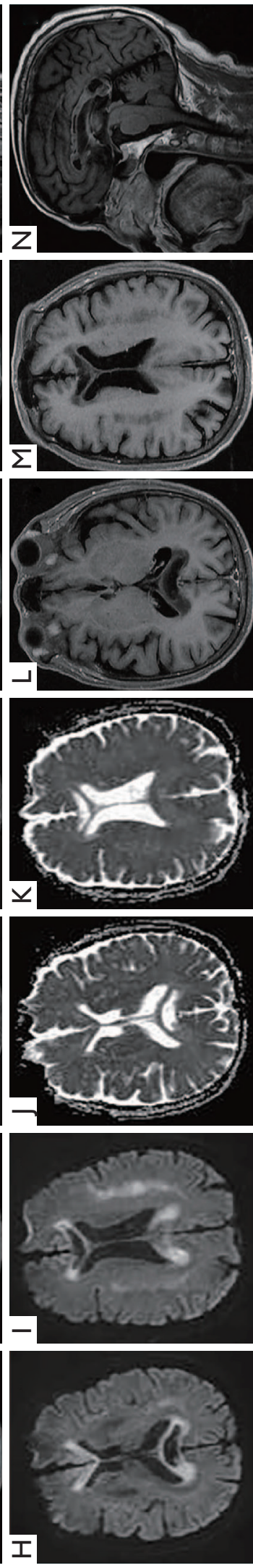
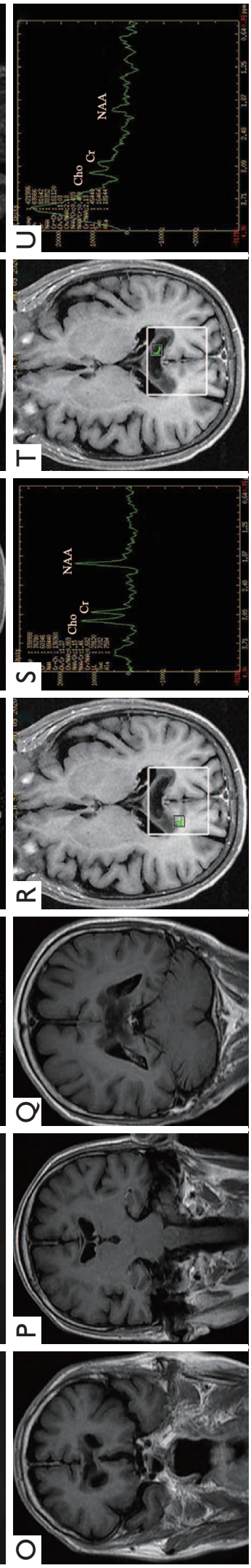

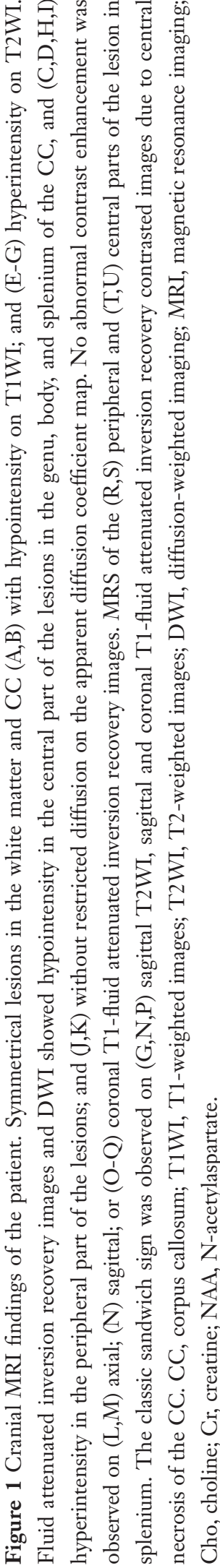


patient with CC lesions in two chronologically distinct stages, a subacute lesion at the rostrum, genu, and body of the CC and an acute lesion in the splenium (2), which may be explained by the progression and recurrence of MBD. The CC lesions of MBD may resemble other inflammatory demyelinating diseases, such as multiple sclerosis, with a progressive demyelination process. Similar to alcoholic MBD, nonalcoholic MBD may also be a recurrent and dynamic pathological process that can eventually cause permanent damage (6).

Furthermore, MBD is related to alcohol abuse and malnutrition, suggesting that the underlying cause is metabolic (3). A generally well-known hypothesis is that toxicity or nutritional deficiencies leads to hypometabolism of the brain and acute demyelination of the CC, and in more severe cases can cause cell necrosis (8). Necrosis and cyst degeneration are especially common in the genu and splenium (9). Moreover, cytokine-mediated cytotoxic edema was considered to be one of the pathophysiological mechanisms of MBD (10). The chronic lesions of our patient were a result of necrosis and cavitation with welldefined margins located in the genu, body, and splenium of the CC. However, when any lesion crosses the $\mathrm{CC}$ with internal necrosis, GBM should also be considered.

GBM is a primary malignancy that is particularly common in the central nervous system and usually extends along white matter tracts, including the CC (11). The typical bilateral hemisphere involvement and CC infiltration of GBM leads to a butterfly pattern (12). The lesions show heterogeneous hypointensity on T1WI and hyperintensity on T2WI, with irregular contrast enhancement, peritumoral vasogenic edema, mass effect, and internal necrosis usually being prominent (12). Cho/NAA ratio of lesions are usually $>2.5$ (13). No abnormal contrast enhancement was observed in the CC of our patient; MRS revealed nonneoplastic lesions, and the final diagnosis was MBD. Furthermore, the application of MRS and gadolinium enhancement could provide additional information that contributes to the differential diagnosis between MBD with necrosis lesions in the CC and GBM.

Brain metabolites are commonly evaluated by MRS, which may provide supplementary information to confirm the diagnosis, monitor disease progression, and inform treatment response (14). In previous MRS evaluations (which are often performed in the acute phase of MBD), the CC was usually included as a region of interest (ROI), and also occasionally the periventricular white matter, right parietal region, and precentral gyrus $(2,9,14-19)$. MRS of the ROI show a reduced NAA peak and NAA/Cr ratio, the Cho and Cho/Cr ratio increase slightly in the acute stage, and a lactate (Lac) peak can be observed $(2,9,14,16,17,19)$.

In the subacute stage, MRS demonstrates a reduction in the NAA/Cr ratio with partial and progressive reduction of the Cho/Cr ratio, and a Lac peak in some cases $(15,16,18)$. Lac typically occurs in the acute and subacute stages of demyelination, during which the lesion switch from aerobic to anaerobic glycolysis $(2,15)$. A reduced $\mathrm{NAA} / \mathrm{Cr}$ ratio is suggestive of neuronal loss secondary to demyelination (15).

In the chronic stage, partial recovery of the $\mathrm{NAA} / \mathrm{Cr}$ ratio and the progressive reduction of the $\mathrm{Cho} / \mathrm{Cr}$ ratio are seen. The temporary dysfunction of axons may explain the partial restoration of the NAA/Cr ratio, and the decreasing $\mathrm{Cho} / \mathrm{Cr}$ ratio is contemporary with the chronic phase of demyelination (15). Lac is replaced by a lipid peak initially in the chronic stage, which is consistent with the major axonal damage. Neither Lac nor lipid peaks are evident at 11 months after the onset of symptoms. MRS findings indicate that metabolites change over time, and support the pathogenetic theory that an inflammatory reaction may be accompanied by demyelination and micronecrosis in MBD (15).

Our MBD patient was without enhancement in the chronic and subacute lesions in the CC. Wang et al. also reported an MBD patient who had undergone repeated MRI scans with gadolinium enhancement in the subacute stage, and necrosis without enhancement had occurred in the former acute lesion with gadolinium enhancement in the splenium (20). A possible explanation for this is that subacute and chronic necrosis lesions of MBD are usually not gadolinium enhanced.

The memory impairment of our patient did not significantly improve, which might have been due to the failure of timely treatment. Also, the CC lesions were necrotic and cystic. He did not respond well to the administration of thiamine supplement, which supports the previous result of delayed treatment being a risk factor for poor outcomes (1). Moreover, the prognosis for MBD patients with CC necrosis is not as good as that for patients in CC demyelination recovery (20).

In conclusion, this is the first report showing that MRS and gadolinium enhancement can provide additional information for assisting in the differential diagnosis between nonalcoholic MBD with necrosis lesions in the CC and GBM. Furthermore, MBD should be suspected in all patients with neuropsychiatric symptoms and an increased risk of malnutrition. We also found two chronologically different phases in our nonalcoholic MBD patient, and 
there were no significant differences in the changing trend of metabolites related to disease stages between alcoholic and nonalcoholic MBD.

\section{Acknowledgments}

We thank the patient and his family for their collaboration. Funding: None.

\section{Footnote}

Conflicts of Interest: All authors have completed the ICMJE uniform disclosure form (available at https://dx.doi. org/10.21037/qims-21-632). The authors have no conflicts of interest to declare.

Ethical Statement: The authors are accountable for all aspects of the work in ensuring that questions related to the accuracy or integrity of any part of the work are appropriately investigated and resolved. All procedures performed in studies involving human participants were in accordance with the ethical standards of the institutional research committee and with the Helsinki Declaration (as revised in 2013). Written informed consent was obtained from the patient for publication of this case report and accompanying images. A copy of the written consent is available for review by the editorial office of this journal.

Open Access Statement: This is an Open Access article distributed in accordance with the Creative Commons Attribution-NonCommercial-NoDerivs 4.0 International License (CC BY-NC-ND 4.0), which permits the noncommercial replication and distribution of the article with the strict proviso that no changes or edits are made and the original work is properly cited (including links to both the formal publication through the relevant DOI and the license). See: https://creativecommons.org/licenses/by-nc-nd/4.0/.

\section{References}

1. Hillbom M, Saloheimo P, Fujioka S, Wszolek ZK, Juvela $\mathrm{S}$, Leone MA. Diagnosis and management of MarchiafavaBignami disease: a review of CT/MRI confirmed cases. J Neurol Neurosurg Psychiatry 2014;85:168-73.

2. Quintas-Neves M, Amorim JM, Soares-Fernandes JP. Marchiafava-Bignami Disease: Two Chronologically Distinct Stages in the Same Patient. Can J Neurol Sci 2020;47:689-90.
3. Rickert CH, Karger B, Varchmin-Schultheiss K, Brinkmann B, Paulus W. Neglect-associated fatal Marchiafava-Bignami disease in a non-alcoholic woman. Int J Legal Med 2001;115:90-3.

4. Arbelaez A, Pajon A, Castillo M. Acute MarchiafavaBignami disease: MR findings in two patients. AJNR Am J Neuroradiol 2003;24:1955-7.

5. Yang L, Liu J, Yin Y, Yu H. Simultaneous acute Marchiafava-Bignami disease and posterior reversible encephalopathy syndrome: a case almost misdiagnosed. Quant Imaging Med Surg 2020;10:1392-5.

6. Li W, Ran C, Ma J. Diverse MRI findings and clinical outcomes of acute Marchiafava-Bignami disease. Acta Radiol 2021;62:904-8.

7. Tao H, Kitagawa N, Kako Y, Yamanaka H, Ito K, Denda K, Koyama T. A case of anorexia nervosa with Marchiafava-Bignami Disease that responded to high-dose intravenous corticosteroid administration. Psychiatry Res 2007;156:181-4.

8. Fiacco F, Barbato L, Pecoraro MG, Maggio P. Vegetarian diet and excessive tea consumption: a dangerous association? Metab Brain Dis 2017;32:271-4.

9. Nalini A, Kovoor JM, Dawn R, Kallur KG. MarchiafavaBignami disease: Two cases with magnetic resonance imaging and positron emission tomography scan findings. Neurol India 2009;57:644-8.

10. Tetsuka S, Hashimoto R. Alcohol-Related Central Nervous System Disorders Associated with Vitamin B Deficiency. SN Compr Clin Med 2021;3:528-37.

11. Cui Y, Zeng W, Jiang H, Ren X, Lin S, Fan Y, Liu Y, Zhao J. Higher Cho/NAA Ratio in Postoperative Peritumoral Edema Zone Is Associated With Earlier Recurrence of Glioblastoma. Front Neurol 2020;11:592155.

12. Park SE, Choi DS, Shin HS, Baek HJ, Choi HC, Kim JE, Choi HY, Park MJ. Splenial Lesions of the Corpus Callosum: Disease Spectrum and MRI Findings. Korean J Radiol 2017;18:710-21.

13. Vallée A, Guillevin C, Wager M, Delwail V, Guillevin R, Vallée JN. Added Value of Spectroscopy to Perfusion MRI in the Differential Diagnostic Performance of Common Malignant Brain Tumors. AJNR Am J Neuroradiol 2018;39:1423-31.

14. Tuntiyatorn L, Laothamatas J. Acute MarchiafavaBignami disease with callosal, cortical, and white matter involvement. Emerg Radiol 2008;15:137-40.

15. Gambini A, Falini A, Moiola L, Comi G, Scotti G. Marchiafava-Bignami disease: longitudinal MR imaging and MR spectroscopy study. AJNR Am J Neuroradiol 
2003;24:249-53.

16. Lee SH, Kim SS, Kim SH, Lee SY. Acute MarchiafavaBignami disease with selective involvement of the precentral cortex and splenium: a serial magnetic resonance imaging study. Neurologist 2011;17:213-7.

17. Consoli A, Pirritano D, Bosco D, Postorino P, Consoli D. Corticosteroid treatment in a patient with MarchiafavaBignami disease. Neurol Sci 2014;35:1143-5.

18. Sarpa MG, Ferrazzoli D, Massa R, Garaci F, Izzi F, Romigi A, Liguori C, Placidi F. Paucisymptomatic MarchiafavaBignami disease with relevant diffusion-weighted MRI

Cite this article as: Zhou Z, Li Q, Pan C, Liang T, Zhang T, $\mathrm{Xu}$ P. Magnetic resonance spectroscopy and gadolinium enhancement assist in the diagnosis of nonalcoholic Marchiafava-Bignami disease with necrosis lesions: a case description. Quant Imaging Med Surg 2022;12(2):1652-1657. doi: 10.21037/qims-21-632 lesions. Int J Neurosci 2013;123:738-40.

19. Machado A, Soares-Fernandes J, Ribeiro M, Rodrigues M, Cerqueira J, Ferreira C. Alcohol abuse and acute behavioural disturbances in a 24-year-old patient. Diagnosis: Marchiafava-Bignami disease (MBD). J Clin Neurosci 2009; 16:811-59.

20. Wang Z, Wang J, Yi F, Zhou L, Zhou Y. Gadolinium Enhancement May Indicate a Condition at Risk of Developing Necrosis in Marchiafava-Bignami Disease: A Case Report and Literature Review. Front Hum Neurosci 2019;13:79. 